\title{
Equivalence of Certain Inequalities Complementing Those of Cauchy-Schwarz and Hölder
}

\author{
J. B. Diaz, ${ }^{1}$ A. J. Goldman, and F. T. Metcalf ${ }^{1}$
}

(August 26, 1964)

\begin{abstract}
An inequality due to the first and third authors, which complements the Cauchy-Schwarz Inequality, is shown equivalent to a result of Rennie. A more general inequality due to the first and third authors, which complements that of Hölder, is proven equivalent to a previously published generalization of Rennie's Inequality.
\end{abstract}

Although the inequalities treated below have discrete analogs $[2 ; 4]^{2}$ as well as Hilbert space operator analogs [6], we shall present only the integral versions. The symbol $I$ will denote integration over a fixed totally finite measure space, the existence and finiteness of the various definite integrals involved being assumed throughout. $M$ and $m$ stand for real numbers with $M>m$.

In [2; 5], Diaz and Metcalf give the following result for real-valued functions $f$ and $g$ with the former nonvanishing: If

$$
m \leqslant g / f \leqslant M
$$

almost everywhere, then

$$
\mathrm{I}\left(\mathrm{g}^{2}\right)+\mathrm{mMI}\left(\mathrm{f}^{2}\right) \leqslant(\mathrm{M}+\mathrm{m}) \mathrm{I}(\mathrm{fg})
$$

with equality if and only if

$$
(\mathrm{M}-\mathrm{g} / \mathrm{f})(\mathrm{g} / \mathrm{f}-\mathrm{m})=0
$$

almost everywhere. Since (2) gives a lower bound for the "inner product" $I(f \mathrm{~g})$ in terms of the squares of the "norms" $\left\{I\left(f^{2}\right)\right\}^{1 / 2}$ and $\left\{I\left(g^{2}\right)\right\}^{1 / 2}$, it can be regarded as a complement to the Cauchy-Schwarz Inequality

$$
I(f g) \leqslant\left\{I\left(f^{2}\right)\right\}^{1 / 2}\left\{I\left(g^{2}\right)\right\}^{1 / 2}
$$

which yields an upper bound for the inner product in terms of the norms.

In [11], Rennie shows for a real-valued function $F$ that if

$$
0<\mathrm{m} \leqslant \mathrm{F} \leqslant \mathrm{M}
$$

\section{almost everywhere, then} ${ }^{1}$ Institute for Fluid Dynamics and Applied Mathematics, University of Maryland; re-
search supported in part by the Air Force Office of Scientific Research-Grant AFOSR $400-64$.

${ }^{2}$ Figures in brackets indicate the literature references at the end of this paper.

$$
\mathrm{I}(\mathrm{F})+\mathrm{MmI}\left(\mathrm{F}^{-1}\right) \leqslant(\mathrm{M}+\mathrm{m}) \mathrm{I}(\mathrm{l})
$$

with equality if and only if

$$
(\mathrm{M}-\mathrm{F})(\mathrm{F}-\mathrm{m})=0
$$

almost everywhere. Our first aim in this note is to exhibit the equivalence between Rennie's Inequality and the one given in the last paragraph.

First, suppose it "given" that (1) implies (2). If $F$ obeys (4), we can set

$$
g=F^{1 / 2}, f=F^{-1 / 2}
$$

in (2) to obtain (5), and can observe that (3) applies if and only if (6) does.

Second, suppose it "given" that (4) implies (5). Let $f$ and $g$ obey (1), and initially assume $m>0$. Then $f_{g}>0$ almost everywhere, so that from the original measure $\mu$ we can obtain a new measure $\mu^{\prime}$ given by

$$
\mu^{\prime}(S)=I\left(f g C_{S}\right)
$$

where $C_{S}$ is the characteristic function of $S$. Let $I^{\prime}$ denote integration over the entire space with respect to $\mu^{\prime}$. Then $f$ and $g$ obey (1) almost everywhere $\left(\mu^{\prime}\right)$ as well as $(\mu)$, so that (4) with $F=g / f$ yields from (5)

$$
I^{\prime}(g / f)+M m I^{\prime}(f / g) \leqslant(M+m) I^{\prime}(1)
$$

which is equivalent to the desired result (2). Moreover equality holds in (2) if and only if it holds almost everywhere $\left(\mu^{\prime}\right)$ in (6), hence iff it holds almost everywhere $\left(\mu^{\prime}\right)$ in (3), thus iff it holds almost everywhere $(\mu)$ in $(3)$.

If the hypothesis $m>0$ is dropped, we need only choose a real number $c$ so large that $m+c>0$, and replace (1) with its consequence

$$
0<m+c \leqslant(g+c f) \mid f \leqslant M+c .
$$


By the case just treated we have

$$
\begin{aligned}
I\left((g+c f)^{2}\right)+(m+c)(M+c) I\left(f^{2}\right) & \leqslant(M+c+m+c) I(f(g+c f))
\end{aligned}
$$

with equality if and only if (3) holds almost everywhere.

The terms in $c$ on both sides of this last inequality (after expansion) are identical, and can be subtracted off to obtain the desired result (2). This completes the proof of equivalence.

Now let $p$ and $q$ be real numbers which are "Hölderconjugate," i.e.,

$$
p>0, q>0, p^{-1}+q^{-1}=1 .
$$

In [3], Diaz and Metcalf announced the discrete ana$\log$ of the following result for a positive-valued function $f$ and a nonnegative-valued function $g$ : If

$$
0 \leqslant m^{p} \leqslant g^{q} / f^{p} \leqslant M^{p}
$$

almost everywhere, then

$$
\begin{aligned}
(M-m)^{-1}\left(M^{p-1}-m^{p-1}\right) I\left(g^{q}\right) & \\
& +(M m)^{p-1} I\left(f^{p}\right) \leqslant(M-m)^{-1}\left(M^{p}-m^{p}\right) I(f g)
\end{aligned}
$$

with equality if and only if

$$
\left(M^{p}-g^{q} / f^{p}\right)\left(g^{q} / f^{p}-m\right)=0
$$

almost everywhere. Since (9) gives a lower bound for the "bilinear product" $I(f g)$ in terms of the "conjugate norms" $\left\{I\left(f^{p}\right)\right\}^{1 / p}$ and $\left\{I\left(g^{q}\right)\right\}^{1 / q}$, it can be regarded as a complement to the Hölder Inequality

$$
I(f g) \leqslant\left\{I\left(f^{p}\right)\right\}^{1 / q}\left\{I\left(g^{q}\right)\right\}^{1 / q}
$$

which yields a corresponding upper bound. Note that (1) and (2) constitute the special case $p=q=2$ of this result.

We shall find it convenient to "normalize" this result by a technique which fails in case $m=0$, and therefore note in advance that an elementary proof is available in case $m=0$ : From (8) we obtain $0 \leqslant g^{q} \leqslant M^{p} f^{p}$, so that

$$
g^{q}=g^{q-1} \cdot g=\left(g^{q}\right)^{(q-1) / q} \cdot g=\left(g^{q}\right)^{1 / p} \cdot g \leqslant M f g
$$

almost everywhere, implying

$$
I\left(g^{q}\right) \leqslant M I(f g)
$$

which is equivalent to (9) with $m=0$; (10) also comes out right.

Assuming now that $m>0$ in (8), we "normalize" by replacing $m$ by $1, M$ by the constant $Q=M / m>1$, and $g$ by the function $h=g / m$. The equivalent normalized version of the result [3] then reads as follows: If $\mathrm{p}>1$, and if the positive-valued functions $\mathrm{f}$ and $\mathrm{h}$ satisfy

$$
1 \leqslant \mathrm{~h} / \mathrm{f} \leqslant \mathrm{Q}
$$

almost everywhere, then

$\left(Q^{p}-Q^{p-1}\right) I\left(f^{p}\right) \leqslant\left(Q^{p}-1\right) I\left(f h^{p-1}\right)-\left(Q^{p-1}-1\right) I\left(h^{p}\right)$

with equality if and only if

$$
(\mathrm{Q}-\mathrm{h} / \mathrm{f})(\mathrm{h} / \mathrm{f}-\mathrm{l})=0
$$

almost everywhere. Here $p>1$ is the necessary and sufficient condition for $p$ to be related to some $q$ as in (7).

In [8], Goldman proved and presented as a normalized generalization of Rennie's Inequality, the following result, which turns out to have been anticipated by Rennie himself (see example 1 of [12]) and also to appear in recent work of A. W. Marshall and I. Olkin [10]: Let $s$ and $r$ be real numbers satisfying

$$
s>r, s r \neq 0 .
$$

If the function $\mathrm{F}$ satisfies

$$
1 \leqslant \mathrm{~F} \leqslant \mathrm{Q}
$$

almost everywhere, then

$$
\begin{aligned}
& \left(Q^{s}-Q^{r}\right) I(1) \leqslant\left(Q^{s}-1\right) I\left(F^{r}\right)-\left(Q^{r}-1\right) I\left(F^{s}\right) \text { if } s r>0 \\
& \left(Q^{s}-Q^{r}\right) I(1) \geqslant\left(Q^{s}-1\right) I\left(F^{r}\right)-\left(Q^{r}-1\right) I\left(F^{s}\right) \text { if } s r<0
\end{aligned}
$$

with equality if and only if

$$
(\mathrm{Q}-\mathrm{F})(\mathrm{F}-\mathrm{l})=0
$$

almost everywhere. (The additional normalization $I(1)=1$ was assumed in [8].) Our second aim is to exhibit the equivalence between this result and the one given in the last paragraph.

First assume it "given" that (11) implies (12). Let $F$ obey (15), and suppose initially that $s>r>0$. Introduce the numbers

$$
p=s /(s-r)>1, \bar{Q}=Q^{s-r}
$$

so that

$$
\bar{Q}^{p}=Q^{s}, \bar{Q}^{p-1}=Q^{r},
$$

and (15) implies that $1 \leqslant F^{s-r} \leqslant \bar{Q}$ almost everywhere. Application of (12) with $\bar{Q}$ replacing $Q, h=F^{s-r}$, and $f=1$, yields (16a) as desired. The argument shows that equality holds in (16a) if and only if the analog

$$
\left(\bar{Q}-F^{s-r}\right)\left(F^{s-r}-1\right)=0
$$

of (13) holds almost everywhere, which is equivalent to (17).

Next suppose $0>s>r$, and set

$$
s^{\prime}=-r, r^{\prime}=-s, \bar{F}=Q F^{-1}
$$


so that

$$
s^{\prime}>r^{\prime}>0,1 \leqslant \bar{F} \leqslant Q .
$$

By the case of (16a) already proved,

$$
\left(Q^{-r}-Q^{-s}\right) I(1) \leqslant\left(Q^{-r}-1\right) I\left(\bar{F}^{-s}\right)-\left(Q^{-s}-1\right) I\left(\bar{F}^{-r}\right) .
$$

i.e.,

$$
\left(Q^{-r}-Q^{-s}\right) I(1) \leqslant\left(Q^{-r}-Q^{-r-s}\right) I\left(F^{r}\right)-\left(Q^{-s}-Q^{-r-s}\right) I\left(F^{s}\right)
$$

which is equivalent to (16a) after multiplication by $Q^{s+r}$. Furthermore, equality holds if and only if

$$
(Q-\bar{F})(\bar{F}-1)=0
$$

almost everywhere, which is seen to be equivalent to (17) after multiplying the first factor by $F Q^{-1}>0$ and the second by $F>0$.

Finally suppose $s>0>r$, and set

$$
s^{\prime}=s-r, r^{\prime}=-r,
$$

so that $s^{\prime}>r^{\prime}>0$. Introduce a new measure $\mu^{\prime}$ (and corresponding integration $I^{\prime}$ ) related to the original measure $\mu$ by

$$
\mu^{\prime}(S)=Q^{r} I\left(F^{r} C_{S}\right) .
$$

Then (15) holds almost everywhere $\left(\mu^{\prime}\right)$ since it holds almost everywhere $(\mu)$; applying the already-proven (16a) with $s^{\prime}$ and $r^{\prime}$ replacing $s$ and $r$ yields

$$
\left(Q^{s-r}-Q^{-r}\right) I^{\prime}(1) \leqslant\left(Q^{s-r}-1\right) I^{\prime}\left(F^{-r}\right)-\left(Q^{-r}-1\right) I^{\prime}\left(F^{s-r}\right),
$$

or equivalently

$$
\left(Q^{s-r}-1\right) I^{\prime}\left(F^{-r}\right) \geqslant\left(Q^{s-r}-Q^{-r}\right) I^{\prime}(1)-\left(1-Q^{-r}\right) I^{\prime}\left(F^{s-r}\right),
$$

which is in turn equivalent to the desired result (16b), the condition for equality also working out correctly.

Second, assume it "given" that (15) implies (16). Suppose that $h$ and $f$ satisfy (11) and that $p>1$. Introduce a new measure $\mu^{\prime}$ (and corresponding integration $\left.I^{\prime}\right)$ related to the original measure $\mu$ by

$$
\mu^{\prime}(S)=I\left(f^{p} C_{S}\right)
$$

Taking $F=h / f$ in (15) with $s=p$ and $r=p-1$ leads via (16a) to
$\left(Q^{p}-Q^{p-1}\right) I^{\prime}(1) \leqslant\left(Q^{p-1}\right) I^{\prime}\left(h^{p-1} / f^{p-1}\right)-\left(Q^{p-1}-1\right) I^{\prime}\left(h^{p} / f^{p}\right)$

which is equivalent to the desired result (12), the condition for equality also working out correctly. This completes the equivalence proof.

Two final remarks are in order. First, a number of known inequalities can be obtained by weakening the ones given here or their discrete or operator analogs, e.g., by applying to one side the relation between arithmetic and geometric means. This particular process when applied to (16) yields the upper bounds for ratios of weighted means obtained by Cargo and Shisha [1], as was noted in [8] and [12]; when applied to (9) it yields an inequality due to Gheorghiu [7], which our results therefore show to be equivalent to the Cargo-Shisha bounds. The specifics for other corollaries are given in the papers cited. Second, the justification for the measure-theoretic maneuvers employed (the transitions between $\mu$ and $I$, and $\mu^{\prime}$ and $I^{\prime}$ ) is supplied by appeal to Halmos [9; p. 134].

\section{References}

[1] G. T. Cargo and O. Shisha, Bounds on ratios of means, J. Res. NBS 66B (Math. and Math. Phys.) No. 4, 169-170 (1962).

[2] J. B. Diaz and F. T. Metcalf, Stronger forms of a class of inequalities of G. Pólya-G. Szegö and L. V. Kantorovich, Bull. Amer. Math. Soc. 69 (1963), 415-418.

[3] J. B. Diaz and F. T. Metcalf, On an inequality complementary to Hölder's inequality, Notices Amer. Math. Soc. 10, No. 5 (8/63), 510.

[4] J. B. Diaz and F. T. Metcalf, Complementary inequalities I: Inequalities complementary to Cauchy's inequality for sums of real numbers, J. Math. Anal. and Appl. 9, 59-74 (1964).

[5] J. B. Diaz and F. T. Metcalf, Complementary inequalities II: Inequalities complementary to the Buniakowsky-Schwarz inequality for integrals, J. Math. Anal. and Appl. 9, 278-293 (1964).

[6] J. B. Diaz and F. T. Metcalf, Complementary inequalities III: Inequalities complementary to Schwarz's inequality in Hilbert space, submitted for publication.

[7] S. A. Gheorghiu, Note sur une Inégalité de Cauchy, Bull. Math. Soc. Roumaine des Sciences 35 (1933), 117-119.

[8] A. J. Goldman, A Generalization of Rennie's Inequality, J. Res. NBS 68B (Math. and Math. Phys.) No. 2, 59-63 (1964).

[9] P. R. Halmos, Measure Theory (van Nostrand, New York, 1950).

[10] A. W. Marshall and I. Olkin, Reversal of the Lyapunov, Hölder, and Minkowski inequalities and other extensions of the Kantorovich inequality, J. Math. Anal. Appl. 8, 503 (1964).

[11] B. C. Rennie, An inequality which includes that of Kantorovich, Amer. Math. Monthly 70 (1963).

[12] B. C. Rennie, On a class of inequalities, J. Australian Math. Soc. 3 (1963) 442-448.

(Paper 68B4-127) 